\title{
Interrelationships between dairy product intake, microflora metabolism, faecal properties and plasmid dissemination in gnotobiotic mice
}

\author{
Sylvie Maisonneuve, Marie-France Ouriet and Yvonne Duval-Iflah* \\ Institut National de la Recherche Agronomique, Centre de Recherche de Jouy en Josas, \\ Unité d'Ecologie et de Physiologie du Système Digestif, Domaine de Vilvert, 78352, Jouy en Josas, cedex, France
}

(Received 30 March 2000 - Revised 12 September 2001 - Accepted 1 October 2001)

\begin{abstract}
We previously described the effects of intake of dairy products on plasmid dissemination in the digestive tract of gnotobiotic mice associated with human faecal flora (HFF) and found that yoghurt, heat-treated yoghurt (HTY) and milk reduced population levels of transconjugants compared with findings in mice fed a standard mouse diet. In the case of lactose intake, transconjugants were not detected. The aim of the present study was to assess the possible interrelationships between these observations and other variables (bacterial ecology, $\mathrm{pH}$, moisture, enzyme activities, short-chain fatty acid (SCFA) contents, lactic acid contents). Much of the interest of the present comparison lies in the fact that the animals were homogeneous in terms of age, gender, food and intestinal microflora, owing to the gnotobiotic mouse model maintained in sterile isolators. We observed no variation in SCFA and lactic acid contents or in the population levels of strictly anaerobic strains of Bacteroides and Bifidobacterium, and of the facultative anaerobic recipient Escherichia coli PG1 strain. The main modifications were the reduction of population levels of transconjugants in mice receiving yoghurt, HTY and milk, and concomitantly an increase of $\beta$-galactosidase and a decrease of $\beta$-glucosidase activities, compared with control mice fed a standard diet. Total inhibition of plasmid transfer was observed in HFF mice consuming lactose, and concomitantly the two enzyme activities ( $\beta$-glucosidase and $\beta$-galactosidase) were increased, compared with the findings in control mice fed a standard diet. In axenic mice consuming lactose, plasmid transfer occurred, $\beta$-galactosidase was not detected and $\beta$-glucosidase was decreased. It is therefore proposed that these two enzyme activities influence plasmid transfer and persistence of transconjugants in the digestive tract of HFF associated mice. When both activities were increased there was a total inhibition of plasmid transfer (case of lactose intake). When $\beta$-galactosidase increased and $\beta$-glucosidase decreased (case of yoghurt, HTY and milk), plasmid transfer occurred at a lower efficiency than in the control group, resulting in lower population levels of transconjugants.
\end{abstract}

Yoghurt: Milk: Lactose: Intestinal metabolism: Plasmid transfer

Plasmid transfer occurs between bacterial strains in the digestive tract of man and animals (Duval-Iflah et al. 1980, 1994; Duval-Iflah \& Chappuis, 1984; Lester et al. 1990). The survival of strains carrying plasmids in the environment depends on many factors. Antibiotic selective pressure is responsible for widespread dissemination of resistance genes in the environment. In the absence of any selective pressure there is an equilibrium between the loss of plasmid (curing) and the plasmid's ability to transfer itself to the cured strains (Duval-Iflah et al. 1980; Duncan et al. 1995). Transconjugants which are formed in the digestive tract are generally at a disadvantage and are rapidly eliminated by intestinal transit, unless antibiotic is administered (Duval-
Iflah et al. 1980). In some instances the transconjugants are capable of colonising the digestive tract of gnotobiotic mice associated with human digestive microflora (Duval-Iflah et al. 1994; Garrigues-Jeanjean et al. 1999). In such experimental mouse models we have shown that some plasmids and transposons, in association with specific recipient strains, give rise to transconjugants able to establish themselves in the digestive tract at high levels in the absence of any selective pressure. The same exceptional animal models were used to assess the effect of dairy products and probiotics on plasmid transfer and on the persistence of transconjugants in the digestive tract. It was shown that consumption of yoghurt lowered the population

\footnotetext{
Abbreviations: CFU, colony-forming units; HTY, heat-treated yoghurt; SCFA, short-chain fatty acids.

* Corresponding author: Dr Y. Duval-Iflah, fax +33 01346524 62, email duval@biotec.jouy.inra.fr
} 
levels of transconjugants by a magnitude of three logs, consumption of milk totally inhibited the establishment of transconjugants, and lactose added to the standard diet prevented their formation (Maisonneuve et al. 2000, 2001).

Beneficial effects of yoghurt and fermented milks are widely described, and in some instances the mechanisms of their action have been proposed. The decrease of diarrhoeic symptoms has been associated with the lowering of intestinal transit, and modification of the composition and metabolism of the microflora (Hotta et al. 1987; Pochart et al. 1989; Marteau et al. 1990; Kaila et al. 1992; Shermak et al. 1995). Antimutagenic effects of dairy products are explained by a decrease in some enzymic activities (Goldin et al. 1980, 1992; Goldin \& Gorbach, 1984a,b; Lidbeck \& Nord, 1991; Bartram et al. 1994), and hypocholesterolemic properties have been correlated with their ability to metabolise cholesterol and bile salt (Gilliland et al. 1985; Gilliland \& Walker, 1990; Walker \& Gilliland, 1993; Buck \& Gilliland, 1994).

The aim of the present work was to study some of the factors known to be modified by dairy products, such as intestinal transit time, composition, metabolic and enzymic activities of the microflora, and moisture and $\mathrm{pH}$ of faeces, in order to detect which of them could be correlated with the lowering of transconjugant densities and the inhibition of plasmid transfer after consumption of yoghurt, milk and lactose.

\section{Materials and methods}

\section{Gnotobiotic mice associated with human faecal flora}

Mice and protocols were those described previously (Maisonneuve et al. 2001). Twenty male adult germ-free $\mathrm{C} 3 \mathrm{H} / \mathrm{He}$ mice (axenic) were obtained from our own experimental facilities and reared in sterile Trexler plastic isolators (La Calhène, Vélizy, France). They were inoculated with $500 \mu \mathrm{l}$ of a $10^{-2}$ dilution of freshly passed faeces from mice colonised with human intestinal microflora containing the recipient Escherichia coli PG1 strain (Garrigues-Jeanjean et al. 1999) with an oesophageal tube, twice at $24 \mathrm{~h}$ intervals. They were fed a sterile standard pelleted mouse diet for 2 weeks (period 1). At the end of period 1, mice were randomised (four per treatment) and transferred to five experimental sterile isolators. Faeces were collected from each mouse in each group, and pooled to give faecal samples for period 1 measurements. Groups were randomised to eat, for 2 weeks, sterile powdered diets that were mixed ( 2 parts diet: 1 part solution) where the solutions were one of the following: water (control); yoghurt; heat-treated yoghurt (HTY); milk; lactose. After 2 weeks of adaptation to the diet (period 2), faeces were collected as in period 1 , and the animals were inoculated with E. coli UB1832 donor strain carrying a selftransmissible plasmid R388. They continued on the same diets for 2 months (period 3). Spores of strictly thermophilic Bacillus subtilis were inoculated together with a donor strain to monitor intestinal transit (Contrepois \& Gouet, 1969). The effects of supplementation on transconjugant formation, on their persistence in the digestive tract and on various factors of interest were evaluated for 2 months (period 3).
Gnotobiotic mice associated with Escherichia coli

Four axenic mice were used to assess the effect of lactose supplementation in the absence of human faecal flora. Axenic mice were transferred into a sterile isolator and received a lactose diet throughout the experiment. During the first $7 \mathrm{~d}$ from the beginning of lactose supplementation, mice were kept axenic. A recipient strain E. coli PG1 and a donor strain $E$. coli UB 1832 were then inoculated at $7 \mathrm{~d}$ intervals. The experiment lasted a further $16 \mathrm{~d}$ after the donor strain was inoculated. Faecal samples were collected in axenic mice: before they received a supplemented diet; $7 \mathrm{~d}$ after lactose adaptation; $7 \mathrm{~d}$ after recipient strain inoculation; $16 \mathrm{~d}$ after donor strain inoculation.

\section{Supplementation}

The yoghurt S85 was obtained with long-life $0 \%$ milk (Finesse $\AA$; SMB, Paris, France), fermented with bacterial symbiosis S85 containing Lactobacillus bulgaricus 100158 and Streptococcus thermophilus 001 158. The HTY was prepared by heating yoghurt $\mathrm{S} 85$ to $70^{\circ} \mathrm{C}$ for $10 \mathrm{~min}$ in a double boiler, then cooling it to $4^{\circ} \mathrm{C}$. The milk was that used to prepare yoghurt S85 and HTY. Batches of yoghurt S85, HTY and milk were provided every 2 weeks by the Centre International de Recherche, Daniel Carasso, Groupe Ferments Fermentation (Le Plessis Robinson, France). For the lactose solution, $4.5 \mathrm{~g}$ lactose $/ 100 \mathrm{ml}$ water was filtered (filter pore size $0.45 \mu \mathrm{m}$; Sarstedt, Nümbrecht, Germany). All products were kept at $4{ }^{\circ} \mathrm{C}$. Throughout the administration period, $10 \mathrm{ml}$ yoghurt, HTY, milk, lactose or water for the control group was mixed daily with $20 \mathrm{~g}$ sterile powdered standard mouse diet (D03, UAR, Villemoissonsur-Orge, France). Animals received autoclaved water to drink ad libitum.

\section{Faecal sampling}

Groups of four mice were housed in separate isolators. Freshly passed faeces were collected and pooled from mice in each group. Four faecal samplings were collected at the end of period 1 (before supplementation), at the end of period 2 (before inoculation of the donor strain and spores) and at the end of period 3. Freshly passed faeces were used immediately after emission for bacterial enumeration and for measurement of $\mathrm{pH}$ and moisture. Other faecal samples were kept at $-80^{\circ} \mathrm{C}$ for further analysis of short-chain fatty acids (SCFA), lactic acid contents and enzymic activities.

\section{pH measurement}

Immediately after emission, the pooled faecal samples were diluted in distilled water $(1: 4, w / v)$ and faecal $\mathrm{pH}$ was determined using a glass $\mathrm{pH}$ electrode (pH-Meter 763 Multi-Calimatic, Knick, Germany).

\section{Faecal moisture}

Freshly passed faeces were used to determine the dry weight with a moisture autoanalyser (MA 30 Sartorius, Goetingen, Germany). 


\section{L-D-lactic acid contents}

These were determined from frozen faecal samples and from dairy products, yoghurt, HTY and milk, with a Technicon autoanalyser (Technicon, Plaisir, France) using an enzymic method.

\section{Short-chain fatty acid contents}

SCFA were extracted from aliquots of frozen faeces and dairy products by two-step extraction, the first with hexane and the second, performed twice, with tert-butylmethylether. SCFA were derivatised as $N(0)$-tert-butyldimethylsilyl ester with $N$-methyl- $N$ (tert-butyldimethylsilyl) trifluoroacetamide. The analyses were performed by GC (Girdel 300; Perichrom Delsi, Longjumeau, France) with a flame ionisation detector. The column is a wide-bore CPSil5CB column (Chrompack, Les Ullis, France; $25 \mathrm{~m} \times$ $0.53 \mathrm{~mm}, 100 \%$ methyl). The carrier gas (He) flow rate was $1 \mathrm{ml} / \mathrm{min}$. The temperature of both the detector and the injector was $300^{\circ} \mathrm{C}$. Oven temperature was programmed to increase from $80^{\circ} \mathrm{C}$ to $280^{\circ} \mathrm{C}$ at a rate of $4^{\circ} \mathrm{C} / \mathrm{min}$; 2-methylpentanoic was used as an internal standard. The extractions and analyses were manual procedures.

\section{Enzymic activities in faeces and dairy products}

Faecal samples were diluted in $0.9 \% \mathrm{NaCl}(1: 19$, w/v). The faecal suspension was homogenised with Ultra Turrax (T25 basic IKA). The homogenate was used for the enzyme assay. The enzyme reaction was run at $37^{\circ} \mathrm{C}$. The reaction contained $0.05 \mathrm{ml}$ homogenate, $0.250 \mathrm{ml}$ sodium phosphate buffer $(0.1 \mathrm{M}, \mathrm{pH} 6.4)$ and $0.1 \mathrm{ml}$ substrate solution $(5 \mathrm{~mm}$, Sigma Chemical Co., St Louis, MO, USA). Assays were carried out of $\alpha$ - and $\beta$-glucosidase, $\alpha$ - and $\beta$-galactosidase, $\beta$-glucuronidase, $\alpha$-fucosidase, $\beta$-xylosidase, $N$-acetyl $\alpha$ and $\beta$-galactosaminidase and $N$-acetyl $\beta$-glucosaminidase, with substrates $p$-nitrophenyl $\alpha$-D- and $\beta$-D-glucopyranoside, $\alpha$-D- and $\beta$-D-galactopyranoside, $\beta$-D-glucuronide, $\alpha$ L-fucopyranoside, $\beta$-D-xylopyranoside, $N$-acetyl $\alpha$-D- and $\beta$-D-galactosaminide and $N$-acetyl $\beta$-D-glucosaminide respectively (Andrieux et al. 1992). The reaction was stopped by adding $0.6 \mathrm{ml} \mathrm{Na} \mathrm{CO}_{3}(0.5 \mathrm{M})$. Readings were taken at $400 \mathrm{~nm}$ with a spectrophotometer (Beckman DU.70, Fullerton, CA, USA). A standard curve was constructed with $p$-nitrophenol (Sigma Chemicals). The protein concentration in each sample used to measure enzymic activities was determined using a method described by Lowry et al. (1951), with bovine serum albumin as the standard. Activities are expressed as nmol/ min per mg protein. All these enzyme activities were also determined in dairy product samples by the same process.

\section{Bacterial enumeration}

Immediately after the mice had defecated, faecal samples were diluted in Liquid Casein Yeast extract medium (Raibaud et al. 1966) and spread onto selective media. For enumeration of strictly anaerobic strains, dilution and inoculated procedures were carried out in an anaerobic chamber with prereduced media. Bacteroides spp. counts were obtained on brain-heart infusion agar (Difco Laboratories, Difco, Becton Dickinson, Le Pont de Claix, France) containing $5 \mathrm{mg}$ haemin per litre (Sigma Chemical Co.) and neomycin (140 mg/l, BHI-Hneo). Inoculated plates were kept in contact with the air for $1 \mathrm{~h}$ (Corthier et al. 1996), then reintroduced into an anaerobic chamber and incubated at $37^{\circ} \mathrm{C}$ for $4 \mathrm{~d}$. A modified Beerens agar medium (Beerens, 1990) containing $35 \mathrm{~g} / \mathrm{l}$ Brain Hart Infusion and $5 \mathrm{~g} / \mathrm{l}$ yeast extract (Difco Laboratories) instead of Columbia medium was used to enumerate Bifidobacterium spp. Plates were incubated anaerobically at $37^{\circ} \mathrm{C}$ for $4 \mathrm{~d}$. Total colonies on each medium were counted. Selective enumeration of E. coli parental strains, recipient PG1 and donor UB1832, and transconjugants carrying the self-transmissible plasmid R388 was performed as previously described (Maisonneuve et al. 2001).

\section{Colony hybridisation screening}

The selectivity of both media, BHI-Hneo agar and modified Beerens agar medium, for the quantification of Bacteroides spp. and Bifidobacterium spp. respectively, was examined. One hundred colonies grown on BHI-Hneo and 100 colonies grown on modified Beerens agar were picked and then placed onto BHI-H agar plates and incubated at $37^{\circ} \mathrm{C}$ for $2 \mathrm{~d}$. All procedures were performed in an anaerobic chamber. The assay was performed in triplicate. Colonies were transferred onto nylon transfer membrane (Amersham, Life Science, Buckinghamshire, UK). The membranes were then treated by serial transfers onto filter papers soaked with denaturation solution for $7 \mathrm{~min}(1.5 \mathrm{M}-\mathrm{NaCl}, 0.5 \mathrm{M}-\mathrm{NaOH})$, and then neutralisation solution twice for $3 \mathrm{~min}$ each time (1.5 M-NaCl, 0.5 M-Tris HCl, pH 7.2, 1 mm-EDTA). They were then washed in $2 \times$ sodium salt citrate, placed on filter papers and fixed using a U.V. cross-linker (Amersham, Life Science). The probes used in the present study are listed in Table 1. They were synthesised by MWG-Biotech AG (Germany) and labelled at their $5^{\prime}$ ends using T4 polynucleotide kinase (GIBCO, BRL, Life Technologies,

Table 1. Oligonucleotide probes and washed temperature $(T)$ used in the present study

\begin{tabular}{|c|c|c|c|c|}
\hline Target group & $\begin{array}{l}\text { Probe } \\
\left(5^{\prime}-3^{\prime}\right)\end{array}$ & $\begin{array}{c}\text { Name } \\
\text { (OPD nomenclature) }\end{array}$ & $\mathrm{T}\left({ }^{\circ} \mathrm{C}\right)$ & Reference \\
\hline Domain bacteria & GCTGCCTCCCGTAGGAGT & S-D-Bact-0338-a-A-18 & 64 & Amann et al. (1990) \\
\hline Bifidobacterium & CGGGTGCTRCCCACTTTCATG & S-G-Bif-1412-a-A-21 & 59.5 & Kaufmann et al. (1997) \\
\hline Bacteroides cluster & GCACTTAAGCCGACACCT & S-*-Bacto-1080-a-A-18 & 60 & Doré et al. (1998) \\
\hline
\end{tabular}

$R$, represents an $A / G$ wobble nucleotide; OPD, oligonucleotide probe database.

For experimental procedures, see p. 122. 
SARL, Eragny, France) and $\left[\gamma^{32}{ }^{32} \mathrm{P}\right] \mathrm{ATP}$ (NEN Life Science Products Inc., Boston, MA, USA). The Bact-0338 probe served as a positive control after use of the specific probe. The membranes were prehybridised with hybridisation buffer (sodium salt citrate $6 \times$, SDS $0.5 \%$, Denhart $2.5 \times$, salmon sperm $125 \mathrm{mg} / \mathrm{l}$ ) for $2 \mathrm{~h}$ at $45^{\circ} \mathrm{C}$ in screw-cap hybridisation tubes. The membranes were hybridised overnight at $45^{\circ} \mathrm{C}$ with hybridisation buffer without Denhart and containing 1 pmol labelled probe $/ \mathrm{ml}$ and washed twice over with sodium salt citrate $2 \times$ and SDS $0.5 \%$ buffer at appropriate temperatures indicated in Table 1 for $30 \mathrm{~min}$. The membranes were visualised by autoradiography by means of electronic radiography (Instant Imager $\Delta$ Packard; Vilbert Lourmat, Marne La Vallée, France) or at $-80^{\circ} \mathrm{C}$, by Kodak X-OmatAR films (Sigma Aldrich, Steinheim, Germany).

\section{Statistical analysis}

The results are expressed as means and standard deviations. The cell counts were transformed to $\log _{10}$ numbers. We compared the average for each period 1,2 and 3, between groups and within each group using Student's $t$ test. We chose to reject the null hypothesis at the $5 \%$ level.

\section{Results}

\section{Bacterial populations}

Bacterial counts were performed in duplicate. The total strictly anaerobic population, approximately $4 \times 10^{10}$ colony-forming units (CFU)/g faeces (data not shown) remained steady in the five groups throughout the three periods. Bacteroides constituted the major bacterial population (10.5 (SD 0.1)) and were confirmed at $94.67 \%$ by colony hybridisation using the Bacteroides-PrevotellaPorphyromonas group-specific probe. Bifidobacteria were at levels 100- to 1000-fold lower than those of Bacteroides (7.5 (SD 0.2)) and were confirmed at $81.33 \%$ by colony hybridisation with the Bifidobacteria-specific probe. Neither Bacteroides nor Bifidobacterium population levels were affected by the dairy product and lactose supplementation (Table 2). The population levels of the recipient strain PG1, approximately $10^{8} \mathrm{CFU} / \mathrm{g}$ faeces, remained steady throughout the experiments in all five groups. The donor strain was transient and eliminated at a faster rate than the transit marker, $24 \mathrm{~h}$ after inoculation. Plasmid R388 was rapidly transferred to the recipient strain $\mathrm{PG} 1$, and the resulting transconjugants (TC-R388) were detected at a low level, approximately $10^{2}$ and $10^{3} \mathrm{CFU} / \mathrm{g}$ faecal samples $6 \mathrm{~h}$ after inoculation of the donor strain, except in faecal samples from mice supplemented with lactose, where they were below the detection threshold of $10^{2} \mathrm{CFU} / \mathrm{g}$ faeces throughout the experiment. During period 3 and at equilibrium, TC-R388 were significantly higher in control group mice $\left(7.3 \log _{10} \mathrm{CFU} / \mathrm{g}\right.$ faeces) than in the groups receiving HTY or yoghurt (respectively 6.6 and $5.2 \log _{10}$ $\mathrm{CFU} / \mathrm{g}$ faeces). In mice supplemented with milk and lactose, the TC-R388 were below the detection threshold of $10^{2}$ CFU/g faeces (Table 2).

\section{Transit time}

Spores of Bacillus used as an intestinal transit marker were at maximal levels $6 \mathrm{~h}$ after their inoculation in all groups except in the mice supplemented with milk, where the maximal level was reached at $12 \mathrm{~h}$, indicating a slower transit time.

\section{Faecal moisture, $\mathrm{pH}$ and lactic acid contents}

No significant variation was observed in moisture, $\mathrm{pH}$ or lactic acid contents in mice fed the control diet, yoghurt, HTY or milk. Mean values ranged from 59.3 (SD 2.0) to 67.1 (SD 2.7) \% in moisture, from 6.05 (SD 0.09) to 6.64 (SD 0.74) in $\mathrm{pH}$, from 4.2 (SD 0.5$)$ to $6.2(\mathrm{SD} 0.2) \mathrm{mmol} / \mathrm{kg}$ faeces in lactic $\mathrm{L}(+)$ acid contents and from $6 \cdot 0$ (SD 0.8 ) to 8.3 (SD 0.4) $\mathrm{mmol} / \mathrm{kg}$ faeces in lactic $\mathrm{D}(-)$ acid contents. Lactic acid contents were similar in the yoghurt and HTY that were used in the present study. The $\mathrm{L}(+)$ isomer constituted $70 \%$ $(7.5 \mathrm{~g} / \mathrm{l} \mathrm{L}(+)$ isomer and $3.8 \mathrm{~g} / \mathrm{l} \mathrm{D}$ isomer). Contents in milk were 1000 -fold lower.

Moisture and $\mathrm{pH}$ were slightly affected by lactose supplementation. The faecal $\mathrm{pH}$ increased from 5.99 (SD 0.15 ) at period 1 to 6.29 (SD 0.20) during periods 2 and 3, and the moisture decreased from 64.5 (SD 2.3) to $59 \cdot 0$ (SD 2.0).

Table 2. Bacterial counts in faecal samples from mice of control group and those fed yoghurt, heat-treated yoghurt (HTY) and milk during period 3 (days 30-60) following inoculation of donor strain of plasmid R388

(Mean values and standard deviations, expressed as $\log _{10}$ colony-forming units/g faeces)

\begin{tabular}{|c|c|c|c|c|c|c|c|c|}
\hline & \multicolumn{2}{|c|}{ Bacteroides } & \multicolumn{2}{|c|}{ Bifidobacteria } & \multicolumn{2}{|c|}{$\begin{array}{l}\text { Recipient } \\
\text { strain PG1 }\end{array}$} & \multicolumn{2}{|c|}{$\begin{array}{c}\text { Transconjugant } \\
\text { PG1 (R388) }\end{array}$} \\
\hline & Mean & $\mathrm{SD}$ & Mean & SD & Mean & SD & Mean & SD \\
\hline Control & $10 \cdot 1$ & 0.7 & $7 \cdot 3$ & 0.6 & $8 \cdot 1$ & 0.3 & $7 \cdot 3$ & 0.3 \\
\hline Yoghurt & $10 \cdot 3$ & 0.4 & $7 \cdot 8$ & 1.4 & $8 \cdot 0$ & 0.1 & $5 \cdot 2^{*}$ & 0.4 \\
\hline HTY & $10 \cdot 3$ & 0.1 & $7 \cdot 8$ & 0.9 & $8 \cdot 2$ & 0.3 & $6 \cdot 6^{*}$ & 0.6 \\
\hline Milk & $9 \cdot 7$ & 0.8 & $7 \cdot 7$ & 0.8 & $8 \cdot 2$ & 0.2 & $<2$ & \\
\hline Lactose & $10 \cdot 1$ & 0.0 & 7.5 & 0.7 & $8 \cdot 1$ & 0.1 & $<2$ & \\
\hline
\end{tabular}

$<2$, below detection threshold of $10^{2} \mathrm{CFU} / \mathrm{g}$ faeces.

Mean values were significantly different from the control values: ${ }^{*} P<0.05$. 
Table 3. Short-chain fatty acids and lactic acid in dairy products and in faecal samples from mice of control, yoghurt, heat-treated yoghurt (HTY) and milk groups, before supplementation (baseline, period 1), during adaptation to supplementation (period 2) and during the day 30 to day 60 period following donor strain inoculation (period 3 )

(Mean values, expressed as $\mathrm{mmol} / \mathrm{kg}$ faeces, with their standard errors)

\begin{tabular}{|c|c|c|c|c|c|c|c|}
\hline & \multirow{2}{*}{$\begin{array}{l}\text { Dairy products } \\
\text { (mg/100 ml) }\end{array}$} & \multicolumn{2}{|c|}{$\begin{array}{l}\text { Period } 1 \\
\text { (baseline) }\end{array}$} & \multicolumn{2}{|c|}{$\begin{array}{c}\text { Period } 2 \\
\text { (adaptation) }\end{array}$} & \multicolumn{2}{|c|}{$\begin{array}{c}\text { Period } 3 \\
\text { (day } 30-\text { day } \\
60 \text { ) }\end{array}$} \\
\hline & & Mean & SE & Mean & SE & Mean & SE \\
\hline \multicolumn{8}{|l|}{ Formic acid } \\
\hline Control & & $7 \cdot 6$ & 0.6 & $7 \cdot 2$ & $3 \cdot 8$ & 8.3 & $2 \cdot 1$ \\
\hline Yoghurt & $30 \cdot 2$ & 9.5 & $2 \cdot 7$ & 9.5 & $5 \cdot 3$ & $7 \cdot 0$ & 4.6 \\
\hline HTY & $17 \cdot 6$ & $8 \cdot 2$ & 1.8 & 8.2 & $2 \cdot 2$ & $7 \cdot 3$ & 0.6 \\
\hline Milk & ND & 8.9 & 3.9 & $7 \cdot 0$ & $1 \cdot 1$ & $7 \cdot 8$ & $1 \cdot 8$ \\
\hline \multicolumn{8}{|l|}{ Acetic acid } \\
\hline Control & & $13 \cdot 7$ & 0.3 & 10.5 & $11 \cdot 6$ & $23 \cdot 7$ & 4.3 \\
\hline Yoghurt & $20 \cdot 5$ & $19 \cdot 3$ & $1 \cdot 7^{\mathrm{a}}$ & $5 \cdot 0$ & $5 \cdot 5^{\mathrm{b}}$ & $16 \cdot 3$ & 5.9 \\
\hline HTY & $18 \cdot 0$ & $16 \cdot 8$ & $2 \cdot 2$ & $16 \cdot 0$ & $3 \cdot 0$ & $22 \cdot 3$ & $1.3^{b, c}$ \\
\hline Milk & $15 \cdot 0$ & $17 \cdot 1$ & $5 \cdot 2$ & $14 \cdot 2$ & $2 \cdot 3$ & 22.5 & $1 \cdot 8^{\mathrm{C}}$ \\
\hline \multicolumn{8}{|c|}{ Propionic acid } \\
\hline Control & & $3 \cdot 3$ & 0.4 & $2 \cdot 2$ & 0.9 & $3 \cdot 7$ & 0.7 \\
\hline Yoghurt & $2 \cdot 4$ & 3.0 & $2 \cdot 4$ & 2.9 & $3 \cdot 2$ & 2.5 & $0.2^{\mathrm{a}}$ \\
\hline HTY & 2.5 & $3 \cdot 7$ & 0.2 & 1.6 & $1 \cdot 3^{b}$ & $4 \cdot 2$ & $0.3^{\mathrm{c}}$ \\
\hline Milk & 0.2 & $2 \cdot 1$ & $1 \cdot 8$ & $2 \cdot 4$ & 0.9 & 5.4 & $1.4^{\mathrm{C}}$ \\
\hline \multicolumn{8}{|c|}{ Iso-butyric acid } \\
\hline Control & & 0.1 & 0.1 & 0.2 & $0 \cdot 1$ & 0.1 & 0.0 \\
\hline Yoghurt & $2 \cdot 7$ & 0.1 & 0.0 & 0.1 & $0 \cdot 0$ & 0.1 & 0.0 \\
\hline HTY & $2 \cdot 7$ & 0.1 & 0.0 & 0.2 & 0.1 & 0.2 & 0.1 \\
\hline Milk & $10 \cdot 3$ & 0.2 & 0.1 & 0.1 & $0 \cdot 1$ & 0.3 & 0.1 \\
\hline \multicolumn{8}{|l|}{ Butyric acid } \\
\hline Control & & 0.2 & 0.0 & 0.4 & 0.2 & 0.2 & 0.1 \\
\hline Yoghurt & $2 \cdot 2$ & 0.9 & $1 \cdot 2$ & 0.1 & $0 \cdot 1$ & 0.1 & 0.1 \\
\hline HTY & 1.0 & 0.2 & 0.1 & 0.2 & 0.1 & 0.2 & 0.1 \\
\hline Milk & 0.5 & 0.2 & 0.1 & $1 \cdot 2$ & 1.6 & 0.2 & 0.0 \\
\hline \multicolumn{8}{|c|}{ Iso-valeric acid } \\
\hline Control & & 0.4 & 0.1 & 0.2 & 0.2 & 0.4 & 0.2 \\
\hline Yoghurt & 0.3 & 0.2 & 0.2 & 0.1 & 0.2 & 0.3 & 0.4 \\
\hline HTY & 0.2 & 0.3 & 0.2 & 0.5 & 0.3 & 1.0 & 0.6 \\
\hline Milk & 0.3 & 0.2 & 0.1 & 1.0 & $1 \cdot 1$ & $1 \cdot 2$ & $0 \cdot 8$ \\
\hline \multicolumn{8}{|l|}{ Valeric acid } \\
\hline Control & & 0.0 & 0.0 & 0.4 & 0.3 & 0.2 & 0.5 \\
\hline Yoghurt & 0.3 & 0.0 & 0.1 & ND & ND & 0.2 & 0.3 \\
\hline HTY & 0.3 & 0.1 & 0.0 & 0.1 & 0.1 & 0.1 & 0.1 \\
\hline Milk & 0.3 & 0.5 & 0.3 & 0.0 & 0.1 & 0.1 & 0.0 \\
\hline \multicolumn{8}{|c|}{ Iso-caproic acid } \\
\hline Control & & 0.0 & 0.1 & 0.1 & 0.1 & 0.1 & 0.0 \\
\hline Yoghurt & 0.6 & 0.1 & 0.1 & ND & ND & 0.1 & 0.1 \\
\hline HTY & 0.4 & 0.1 & 0.0 & 0.0 & 0.0 & 0.1 & 0.1 \\
\hline Milk & ND & 0.2 & 0.2 & 0.1 & 0.1 & 0.1 & 0.0 \\
\hline \multicolumn{8}{|c|}{ Caproic acid } \\
\hline Control & & 0.1 & 0.17 & $0 \cdot 1$ & 0.0 & 0.2 & 0.0 \\
\hline Yoghurt & $1 \cdot 2$ & 0.2 & 0.2 & 0.1 & 0.0 & 0.2 & 0.0 \\
\hline HTY & $1 \cdot 3$ & 0.1 & 0.0 & 0.1 & $0 \cdot 0$ & 0.2 & 0.0 \\
\hline Milk & 11.4 & 0.1 & 0.0 & 0.1 & 0.0 & 0.2 & 0.0 \\
\hline \multicolumn{8}{|c|}{ Heptanoic acid } \\
\hline Control & & 0.1 & 0.0 & 0.3 & $0 \cdot 3$ & 0.1 & $0 \cdot 1$ \\
\hline Yoghurt & ND & 0.3 & 0.3 & 0.6 & 0.6 & 0.1 & 0.1 \\
\hline HTY & ND & 0.2 & 0.1 & 0.5 & 0.5 & 0.2 & 0.1 \\
\hline Milk & ND & 0.2 & 0.2 & 0.1 & 0.1 & 0.3 & 0.2 \\
\hline
\end{tabular}

ND, not detected.

aValues significantly different from control values in the same period $(P<0.05)$.

${ }^{b}$ Values significantly different from baseline values in the same group $(P<0.05)$.

${ }^{\mathrm{C}}$ Values significantly different from period 2 values in the same group $(P<0.05)$.

For experimental procedures, see p. 123. 
Table 4. Bacterial enzyme activities in faecal samples from mice in the control group, and groups fed yoghurt, heat-treated yoghurt (HTY) or milk before supplementation (baseline, period 1), during adaptation to supplementation (period 2) and during the day 30 to day 60 period following donor strain inoculation (period 3 )

(Mean values, expressed as $\mathrm{nmol} / \mathrm{min}$ per $\mathrm{mg}$ protein, and standard deviations)

\begin{tabular}{|c|c|c|c|c|c|c|}
\hline & \multicolumn{2}{|c|}{$\begin{array}{l}\text { Period } 1 \\
\text { (baseline) }\end{array}$} & \multicolumn{2}{|c|}{$\begin{array}{c}\text { Period 2 } \\
\text { (adaptation) }\end{array}$} & \multicolumn{2}{|c|}{$\begin{array}{l}\text { Period } 3 \text { (day } \\
30-\text { day } 60 \text { ) }\end{array}$} \\
\hline & Mean & SD & Mean & SD & Mean & SD \\
\hline \multicolumn{7}{|c|}{$\alpha$-Galactosidase } \\
\hline Control & 105.6 & $1 \cdot 8$ & 38.6 & $26 \cdot 4^{\mathrm{b}}$ & $60 \cdot 8$ & 21.6 \\
\hline Yoghurt & 89.3 & 3.4 & $77 \cdot 1$ & $10 \cdot 0^{\mathrm{a}}$ & 74.8 & $13 \cdot 3$ \\
\hline HTY & $61 \cdot 6$ & $6 \cdot 9^{\mathrm{a}}$ & $81 \cdot 3$ & 32.6 & $102 \cdot 1$ & 22.7 \\
\hline Milk & $45 \cdot 9$ & $6 \cdot 8^{\mathrm{a}}$ & $57 \cdot 2$ & $11 \cdot 3$ & $102 \cdot 1$ & $65 \cdot 16$ \\
\hline \multicolumn{7}{|c|}{$\beta$-Galactosidase* } \\
\hline Control & $75 \cdot 3$ & $14 \cdot 8$ & $20 \cdot 6$ & $8 \cdot 1$ & $48 \cdot 3$ & 13.5 \\
\hline Yoghurt & 55.4 & $37 \cdot 1$ & $45 \cdot 6$ & $14 \cdot 3$ & 124.5 & $36 \cdot 1^{a, b}$ \\
\hline HTY & 58.4 & $19 \cdot 7$ & 38.9 & 5.9 & $130 \cdot 7$ & $39 \cdot 2^{a, b}$ \\
\hline Milk & $43 \cdot 1$ & 3.5 & $54 \cdot 7^{\mathrm{a}}$ & $2 \cdot 6^{\mathrm{a}}$ & $135 \cdot 2^{\mathrm{b}}$ & $39 \cdot 4^{a, b}$ \\
\hline Lactose & $27 \cdot 6$ & 11.0 & & & $84 \cdot 3$ & $15 \cdot 4^{a, b}$ \\
\hline \multicolumn{7}{|c|}{$\alpha$-Glucosidase } \\
\hline Control & 24.5 & 18.5 & $20 \cdot 2$ & $4 \cdot 1$ & 39.5 & 23.2 \\
\hline Yoghurt & 54.0 & 11.5 & $27 \cdot 2$ & $9 \cdot 1$ & $26 \cdot 7$ & 2.5 \\
\hline HTY & $46 \cdot 9$ & $12 \cdot 6$ & $34 . \overline{5}$ & $6 \cdot 4^{\mathrm{a}}$ & 32.2 & $7 \cdot 0$ \\
\hline Milk & $46 \cdot 5$ & $10 \cdot 6$ & 28.4 & $7 \cdot 8$ & $27 \cdot 2$ & $6 \cdot 6^{\mathrm{b}}$ \\
\hline \multicolumn{7}{|c|}{$\beta$-Glucosidase } \\
\hline Control & $42 \cdot 2$ & $2 \cdot 0$ & 11.9 & $8 \cdot 1^{b}$ & $31 \cdot 1$ & $12 \cdot 8$ \\
\hline Yoghurt & $30 \cdot 7$ & $3 \cdot 8$ & $21 \cdot 6$ & 8.5 & $12 \cdot 2$ & $4 \cdot 6^{a, b}$ \\
\hline HTY & 34.7 & 4.6 & 24.5 & $7 \cdot 6$ & $12 \cdot \overline{6}$ & 11.0 \\
\hline Milk & $29 \cdot 3$ & 4.2 & $20 \cdot 0$ & $5 \cdot 7$ & $10 \cdot 7$ & $7 \cdot 8^{a, b}$ \\
\hline Lactose & $24 \cdot 2$ & $5 \cdot 50$ & & & 54.9 & $4 \cdot 7^{\mathrm{a}, \mathrm{b}}$ \\
\hline \multicolumn{7}{|c|}{$\beta$-Glucuronidase } \\
\hline Control & 11.4 & 2.6 & $7 \cdot 3$ & $5 \cdot 3$ & $13 \cdot 8$ & 3.6 \\
\hline Yoghurt & $12 \cdot 8$ & $1 \cdot 0$ & $7 \cdot 6$ & $5 \cdot 2$ & $7 \cdot 0$ & $0.4^{a, b}$ \\
\hline HTY & $8 \cdot 8$ & 1.6 & $6 \cdot 7$ & 2.8 & $5 \cdot 8$ & $1.4^{\mathrm{a}}$ \\
\hline Milk & $7 \cdot 7$ & 1.49 & $5 \cdot 8$ & 3.5 & $7 \cdot 2$ & $1.3^{\mathrm{a}}$ \\
\hline \multicolumn{7}{|c|}{$\beta$-Xylosidase } \\
\hline Control & $7 \cdot 7$ & 1.0 & 1.7 & $1 \cdot 6^{\mathrm{b}}$ & $7 \cdot 0$ & $2 \cdot 8^{\mathrm{b}}$ \\
\hline Yoghurt & $7 \cdot 0$ & 0.0 & $4 \cdot 7$ & $2 \cdot 3$ & $5 \cdot 2$ & 2.5 \\
\hline HTY & $6 \cdot 2$ & 1.7 & $6 \cdot 0$ & $2 \cdot 4^{\mathrm{a}}$ & $5 \cdot 4$ & 1.5 \\
\hline Milk & $5 \cdot 3$ & 0.2 & 3.4 & $2 \cdot 3$ & 5.4 & 1.2 \\
\hline \multicolumn{7}{|c|}{$\alpha$-Fucosidase } \\
\hline Control & $6 \cdot 1$ & $3 \cdot 1$ & 1.0 & $0.6^{b}$ & 4.0 & $1.6^{\mathrm{b}}$ \\
\hline Yoghurt & $6 \cdot 1$ & $2 \cdot 8$ & 1.9 & $1 \cdot 1^{\mathrm{b}}$ & 3.0 & 0.4 \\
\hline HTY & $2 \cdot 8$ & 0.0 & 2.9 & $0 \cdot 8^{\mathrm{a}}$ & $4 \cdot 2$ & 1.3 \\
\hline Milk & 1.5 & $0.5^{\mathrm{a}}$ & $1 \cdot 7$ & 0.9 & $4 \cdot 1$ & $1 \cdot 2^{\mathrm{b}}$ \\
\hline \multicolumn{7}{|c|}{$N$-acetyl- $\alpha$-galactosaminidase } \\
\hline Control & 0.5 & 0.4 & 0.3 & 0.1 & 0.9 & 0.4 \\
\hline Yoghurt & 0.7 & 0.4 & 0.9 & 0.1 & 0.2 & 0.2 \\
\hline HTY & $0 \cdot 1$ & $0 \cdot 1$ & 0.9 & $0 \cdot 1^{b}$ & 0.4 & 0.5 \\
\hline Milk & 0.2 & 0.3 & 0.6 & 0.2 & 0.5 & 0.0 \\
\hline \multicolumn{7}{|c|}{$N$-acetyl- $\beta$-galactosaminidase } \\
\hline Control & 11.9 & $6 \cdot 3$ & 5.6 & 2.4 & 9.9 & $4 \cdot 1$ \\
\hline Yoghurt & 11.9 & $6 \cdot 7$ & $12 \cdot 3$ & 1.7 & $1 \cdot 0$ & $1.9^{a, b}$ \\
\hline HTY & 6.5 & $5 \cdot 2$ & $11 \cdot 8$ & $0 \cdot 1$ & $9 \cdot 3$ & $3 \cdot 4$ \\
\hline Milk & 4.9 & $5 \cdot 1$ & 7.9 & $1 \cdot 2$ & $7 \cdot 1$ & 4.4 \\
\hline \multicolumn{7}{|c|}{$N$-acetyl- $\beta$-glucosaminidase } \\
\hline Control & $107 \cdot 8$ & $13 \cdot 5$ & $35 \cdot 2$ & $191 \cdot 1^{b}$ & 74.2 & $8 \cdot 0^{\mathrm{b}}$ \\
\hline Yoghurt & $110 \cdot 7$ & $10 \cdot 4$ & $54 \cdot 3$ & $32 \cdot 5$ & $2 \cdot 0$ & $2 \cdot 0^{\mathrm{a}, \mathrm{b}}$ \\
\hline HTY & 65.0 & $23 \cdot 6$ & 59.4 & 14.7 & $43 \cdot 3$ & 8.5 \\
\hline Milk & 44.4 & $10 \cdot 3^{a}$ & 48.0 & 24.7 & 41.9 & 14.1 \\
\hline
\end{tabular}

${ }^{a}$ Values were significantly different from control values at the same period $(P<0.05)$.

${ }^{b}$ Values were significantly different from baseline values in the same group $(P<0.05)$.

${ }^{*} \beta$-Galactosidase was detected in yoghurt at 21.0 (SD 2.0). The other activities were not detected in the dairy products.

For experimental procedures, see p. 123.

\section{Short-chain fatty acids}

The mean concentrations ( $\mathrm{mmol} / \mathrm{kg}$ faeces) of the ten SCFA analysed were similar in all groups and were not modified after supplementation with the dairy products, during periods 2 and 3 (Table 3 ). The distribution and concentrations of SCFA $(\mathrm{mg} / 100 \mathrm{ml})$ in yoghurt and HTY were similar. Heptanoic $\left(\mathrm{C}_{7}\right)$ acid was not detected in any of the dairy products and formic $\left(\mathrm{C}_{1}\right)$ and isocaproic $\left(\mathrm{iC}_{6}\right)$ acids were not detected in milk. Isobutyric $\left(\mathrm{iC}_{4}\right)$ and $\mathrm{n}$-caproic $\left(\mathrm{C}_{6}\right)$ acids were at higher concentrations in milk.

\section{Enzyme activities}

Enzyme activities (Table 4) are expressed as nmol/min per $\mathrm{mg}$ protein. Modifications were observed during periods 2 and 3 . The $\beta$-galactosidase activity was increased in mice of the four groups (124.5 (SD 36.1) v. 55.4 (SD 37.1) in the group receiving yoghurt, 130.7 (SD 39.2) v. 58.4 (SD 19.8) in the group receiving HTY, 135.2 (SD 39.4) v. 43.1 (SD 3.5) in the group receiving milk and 84.3 (SD 15.4) v. 27.6 (SD 11.0) in the group receiving lactose). The $\beta$-glucosidase decreased in the yoghurt, milk, and HTY groups (from 30.7 (SD 3.8) to 12.2 (SD 4.6) in the yoghurt group, from 29.4 (SD 4.2) to 10.7 (SD 7.8) in the milk group and from 34.7 (SD 4.6 ) to 12.6 (SD 10.9) in the HTY group), and increased in the lactose group (from 24.2 (SD 5.5) to 54.9 (SD 4.8)). The $\alpha$-galactosidase activity increased in the milk and HTY groups. The $N$-acetyl- $\beta$-glucosaminidase and $N$-acetyl- $\beta$ galactosaminidase decreased in the group receiving yoghurt.

\section{Effect of lactose intake on plasmid transfer and on $\beta$ - galactosidase and $\beta$-glucosidase activities in axenic mice}

Axenic mice supplemented with lactose were inoculated with recipient and donor strains at $7 \mathrm{~d}$ intervals. The recipient strain became established rapidly after its inoculation and reached a steady state throughout the mating experiment at a mean value of 9.1 (SD 0.2) log $\mathrm{CFU} / \mathrm{g}$ faeces. The donor strain became established at 6.5 (SD 0.2) $\log \mathrm{CFU} / \mathrm{g}$ faeces from day 14 to day 30 . Transconjugant TC-R388 appeared at $4.9 \log \mathrm{CFU} / \mathrm{g}$ faeces, $3 \mathrm{~h}$ after inoculation of the donor strain, then its level increased progressively to reach a maximal level of 9.7 (SD $0 \cdot 2) \log \mathrm{CFU} / \mathrm{g}$ faeces.

There was no $\beta$-galactosidase activity in faecal material of axenic mice before or after lactose supplementation, or in mice associated with $E$. coli strains and supplemented with lactose. This activity was detected at 26.4 (SD 2.5) $\mathrm{nmol} / \mathrm{min}$ per $\mathrm{mg}$ protein only after $30 \mathrm{~d}$ lactose supplementation and after transconjugants were at their maximal density.

$\beta$-glucosidase was detected at 2.4 (SD 0.3 ) in faeces of axenic mice before lactose supplementation, then decreased significantly to 0.5 (SD $0 \cdot 1$ ) after $7 \mathrm{~d}$ lactose supplementation and increased to 1.7 (SD 0.2) $7 \mathrm{~d}$ after the recipient $E$. coli strain was inoculated. It decreased again to $1 \cdot 0$ (SD $0 \cdot 1) 16 \mathrm{~d}$ after the donor strain was inoculated and transconjugants established at a high population level. 


\section{Discussion}

The main objective of the present study was simultaneously to investigate the effects of yoghurt, HTY, milk and lactose consumption on the formation of transconjugants and their persistence in the digestive tract, and look for a correlation with bacterial ecology, metabolism, enzyme activities, $\mathrm{pH}$ and moisture. Much of the interest of the present comparison lay in the fact that the animals were homogeneous in terms of age, gender, food and intestinal microflora, owing to the use of a gnotobiotic mouse model in sterile isolators. Use of such a procedure makes it possible to reveal any relationship between diet and potential modifications of the entities studied.

Our results are consistent with other studies performed in healthy subjects or animals exhibiting stability in terms of faecal moisture, $\mathrm{pH}$ and total anaerobic bacteria after the consumption of various dairy products including fermented milks, heat-treated fermented milks or milk (Goldin \& Gorbach, 1984a,b; Bartram et al. 1994; Pedrosa et al. 1995; Bouhnik et al. 1996; Guerin-Danan et al. 1998; Chen et al. 1999). Our data showed that consuming dairy products or lactose did not result in any modification of the composition of the flora as far as the strictly anaerobic fraction (Bacteroides and Bifidobacteria) and facultative anaerobic Enterobacteria, represented by the recipient E. coli PG1 strain, were concerned (Table 2). The main modification was the reduction of the population level of transconjugants in the yoghurt, HTY and milk groups, and inhibition of transfer in the lactose group. In order for a transconjugant to establish itself in the environment (including the digestive tract), the plasmid has to maintain itself in the recipient strain. In the absence of any selective pressure, an equilibrium is established between the loss of the plasmid (curing) and its ability to transfer itself to the cured strains (Duncan et al. 1995). A low population level of transconjugants can result from an imbalance between these two steps (Duval-Iflah et al. 1980). Our observations concerning the decrease of the population densities of transconjugants after the mice had received yoghurt, HTY and milk can be explained by such an imbalance. We can speculate that the three dairy products either promoted the loss of the plasmid or reduced the efficiency of plasmid transfer, or influenced both steps. Lactose can be implicated, earlier, at the first step of the mating, that allows the formation of transconjugants, since these were not detected in faecal samples.

What can be the factors that might inhibit plasmid transfer in vivo? Do metabolites, $\mathrm{pH}$, moisture or enzyme activities influence or inhibit plasmid transfer? These questions have not previously been answered.

Moisture and $\mathrm{pH}$ were shown in the present study to be exclusively modified by lactose, but to a very small extent for being considered responsible for inhibition of plasmid transfer. Lactic acid and SCFA contents were not affected by the diets.

Only enzyme activities were shown to be affected by the diets (Table 4). Yoghurt, HTY and milk increased $\beta$ galactosidase and decreased $\beta$-glucosidase. Lactose was distinguished by increasing both activities. It is not clear whether those modifications were responsible for inhibition of transfer. Data on the transfer of plasmids in axenic mice were of great interest, and showed that lactose, in the absence of complex human flora, did not inhibit plasmid transfer or the colonisation ability of transconjugants, which became established at the maximum population level, indicating an efficiency of transfer (ratio of 1 between transconjugants and recipients).

$\beta$-Galactosidase was not detected in faecal samples of axenic mice before lactose supplementation, confirming the absence of this activity in adult germ-free mice. This activity was not induced after $7 \mathrm{~d}$ lactose intake or after recipient, donor and transconjugant strains became established in mice consuming lactose. The $\beta$-galactosidase activity was detected only after $30 \mathrm{~d}$ lactose supplementation, at a low level of 26.4 (SD 2.5), which was similar to that found in the control group of mice inoculated with human faecal flora and fed the standard diet, and where transfer and colonisation with transconjugants were efficient and maximal (Tables 2 and 4). Therefore, we propose that inhibition of plasmid transfer is directly correlated with the augmentation of $\beta$-galactosidase activity in all groups of mice inoculated with human faecal flora and supplemented with yoghurt, HTY, milk or lactose (Tables 2 and 4). In mice inoculated with human faecal flora and ingesting lactose, the $\beta$-glucosidase activity was increased, whereas it was decreased in those mice receiving yoghurt, HTY or milk, and in axenic mice receiving lactose supplementation. We suggest that simultaneous augmentation of both $\beta$ galactosidase and $\beta$-glucosidase activities contributed to the total inhibition of transfer in the mice inoculated with human faecal flora and receiving lactose, and that augmentation of $\beta$-galactosidase followed by decrease of $\beta$-glucosidase partially inhibited this transfer in the groups receiving yoghurt and HTY. The case of mice supplemented with milk was particular, since the mating was observed some hours (6) after the donor was inoculated in mice and the transconjugants were around $10^{2} \mathrm{CFU} / \mathrm{g}$ faeces, but they were eliminated from the digestive tract after $24 \mathrm{~h}$ (Maisonneuve et al. 2001).

The mechanisms of action of plasmid transfer and persistence of transconjugants are complex and involve multiple factors. In vitro studies have suggested that inhibition of plasmid transfer might be obtained by chemical agents that affect the formation of sex pili and plasmid curing, such as SDS, organic solvents, acridine dyes, high temperature and proteases (Hahn 1976; Viljanen \& Boratynski, 1991). The present study indicated that some enzyme activities could affect plasmid transfer in vivo in the digestive tract. Further investigation is necessary to evaluate the effects of other substrates on these activities and on plasmid transfer.

Taken together, the present results indicated concomitant effects of the four dairy products on two enzymic activities ( $\beta$-glucosidase and $\beta$-galactosidase) and plasmid transfer. However, it is difficult to establish a relationship between the effect of diet on faecal bacteria and their metabolism in the intestine. Most metabolites are rapidly absorbed through the colonic mucosa after their formation. Therefore, their concentrations in faecal samples do not reflect those in the caecum and proximal colon. In the present study, we monitored the kinetics of the implantation of transconjugants, 
the efficiency of plasmid transfer and the evolution of biochemical and bacteriological factors in faecal samples. It would be informative to perform similar measurements in different digestive tract compartments after a long period of chronic supplementation.

\section{Conclusion}

It is probable that our observations on plasmid dissemination in the digestive tract of mice inoculated with human faecal flora were related to multiple factors, including enzyme activities. These results are of interest and should be pursued in order to find a substrate that can be used as a nutrient to prevent plasmid transfer in the digestive tract and which can compete with the selective pressure exerted by antibiotics during antibiotherapy.

\section{Acknowledgements}

The present study was supported by the Mission Scientifique de SYNDIFRAIS. The authors wish to thank M. C. Degivry for providing dairy products, C. Andrieux, P. Vaissade and N. Khélifa for their assistance in the enzymic and chromatographic analyses and A. M. Wall for revising the English version of the manuscript.

\section{References}

Amann RI, Binder BJ, Olson RJ, Chisholm SW, Devereux R \& Stahl DA (1990) Combination of 16S rRNA-targeted oligonucleotide probes with flow cytometry for analyzing mixed microbial populations. Applied and Environmental Microbiology 56, 1919-1925.

Andrieux C, Pacheco ED, Bouchet B, Gallant D \& Szylit O (1992) Contribution of the digestive tract microflora to amylomaize starch degradation in the rat. British Journal of Nutrition 67, 489-499.

Bartram HP, Scheppach W, Gerlach S, Ruckdeschel G, Kelber E \& Kasper H (1994) Does yogurt enriched with Bifidobacterium longum affect colonic microbiology and fecal metabolites in health subjects? American Journal of Clinical Nutrition 59, $428-432$.

Beerens H (1990) An elective and selective isolation medium for Bifidobacterium spp. Letters in Applied Microbiology 11, $155-157$.

Bouhnik Y, Flourie B, Andrieux C, Bisetti N, Briet F \& Rambaud JC (1996) Effects of Bifidobacterium sp fermented milk ingested with or without inulin on colonic bifidobacteria and enzymatic activities in healthy humans. European Journal of Clinical Nutrition 50, 269-273.

Buck LM \& Gilliland SE (1994) Comparisons of freshly isolated strains of Lactobacillus acidophilus of human intestinal origin for ability to assimilate cholesterol during growth. Journal of Dairy Science 77, 2925-2933.

Chen RM, Wu JJ, Lee SC, Huang AH \& Wu HM (1999) Increase of intestinal Bifidobacterium and suppression of coliform bacteria with short-term yogurt ingestion. Journal of Dairy Science 82, 2308-2314.

Contrepois M \& Gouet P (1969) Utilisation d'une technique microbiologique pour la mesure de la vitesse des microparticules dans le tractus digestif des ruminants (Use of a microbiologic technique for the measurement of transit time of microparticles in the digestive tract of ruminants). Comptes Rendus de l'Académie des Sciences, Paris 268, 1757-1759.
Corthier G, Muller MC \& L'Haridon R (1996) Selective enumeration of Bacteroides vulgatus and B. distasonis organisms in the predominant human fecal flora by using monoclonal antibodies. Applied and Environmental Microbiology 62, 735-738.

Doré J, Sghir A, Hannequart-Gramet G, Corthier G \& Pochart P (1998) Design and evaluation of a $16 \mathrm{~S}$ rRNA-targeted oligonucleotide probe for specific detection and quantitation of human faecal Bacteroides populations. Systematic and Applied Microbiology 21, 65-71.

Duncan KE, Ferguson N \& Istock CA (1995) Fitnesses of conjugative plasmid and its host bacteria in soil microcosms. Molecular Biology and Evolution 12, 1012-1021.

Duval-Iflah Y \& Chappuis JP (1984) Influence of plasmids on the colonization of the intestine by strains of Escherichia coli in gnotobiotic and conventional animals. International Symposium on Microbial Ecology, East Lansing, MI, USA;1983/08/07-12. In Current Perspectives in Microbial Ecology. Proceedings, pp. 264-272 [MJ Klug and CA Reddy, editors]. Washington, DC: American Society for Microbiology.

Duval-Iflah Y, Gainche I, Ouriet MF, Lett MC \& Hubert JC (1994) Recombinant DNA transfer to Escherichia coli of human faecal origin in vitro and in the digestive tract of gnotobiotic mice. FEMS Microbiology Ecology 15, 79-88.

Duval-Iflah Y, Raibaud P \& Rousseau M (1981) Antagonisms among isogenic strains of Escherichia coli in the digestive tracts of gnotobiotic mice. Infection and Immunity 34, 957-969.

Duval-Iflah Y, Raibaud P, Tancrede C \& Rousseau M (1980) R-plasmid transfer from Serratia liquefaciens to Escherichia coli in vitro in the digestive tract of gnotobiotic mice associated with human fecal flora. Infection and Immunity 28, 981-990.

Garrigues-Jeanjean N, Wittmer A, Ouriet MF \& Duval-Iflah Y (1999) Transfer of the shuttle vector pRRI207 between Escherichia coli and Bacteroides spp. in vitro and in vivo in the digestive tract of axenic mice and in gnotoxenic mice inoculated with a human microflora. FEMS Microbiology Ecology 29, 33-43.

Gilliland SE, Nelson CR \& Maxwell C (1985) Assimilation of cholesterol by Lactobacillus acidophilus. Applied and Environmental Microbiology 49, 377-381.

Gilliland SE \& Walker DK (1990) Factors to consider when selecting a culture of Lactobacillus acidophilus as a dietary adjunct to produce a hypocholesterolemic effect in humans. Journal of Dairy Science 73, 905-911.

Goldin BR \& Gorbach SL (1984a) Alterations of the intestinal microflora by diet, oral antibiotics, and Lactobacillus: decreased production of free amines from aromatic nitro compounds, azo dyes, and glucuronides. Journal of the National Cancer Institute 73, 689-695.

Goldin BR \& Gorbach SL (1984b) The effect of milk and lactobacillus feeding on human intestinal bacterial enzyme activity. American Journal of Clinical Nutrition 39, 756-761.

Goldin BR, Gorbach SL, Saxelin M, Barakat S, Gualtieri L \& Salminen S (1992) Survival of Lactobacillus species (strain GG) in human gastrointestinal tract. Digestive Diseases and Sciences 37, $121-128$.

Goldin BR, Swenson L, Dwyer J, Sexton M \& Gorbach SL (1980) Effect of diet and Lactobacillus acidophilus supplements on human fecal bacterial enzymes. Journal of the National Cancer Institute 64, 255-261.

Guerin-Danan C, Chabanet C, Pedone C, Popot F, Vaissade P, Bouley C, Szylit O \& Andrieux C (1998) Milk fermented with yogurt cultures and Lactobacillus casei compared with yogurt and gelled milk: influence on intestinal microflora in healthy infants. American Journal of Clinical Nutrition 67, 111-117.

Hahn FE (1976) Experimental elimination of R factors. Antibiotics Chemotherapy 20, 196-226. 
Hotta M, Sato Y, Iwata S, Yamashita N, Sunakawa K \& Oikawa T et al. (1987) Clinical effects of Bifidobacterium preparations on pediatric intractable diarrhea. Keio Journal of Medecine 36, $298-314$

Kaila M, Isolauri E, Soppi E, Virtanen E, Laine S \& Arvilommi H (1992) Enhancement of the circulating antibody secreting cell response in human diarrhea by a human Lactobacillus strain. Pediatric Research 32, 141-144.

Kaufmann P, Pfefferkorn A, Teuber M \& Meile L (1997) Identification and quantification of Bifidobacterium species isolated from food with genus-specific 16S rRNA-targeted probes by colony hybridization and PCR. Applied and Environmental Microbiology 63, 1268-1273.

Lester SC, del Pilar Pla M, Wang F, Perez-Shael I, Jiang H \& O'Brien TH (1990) The carriage of Escherichia coli resistant to antimicrobial agents by healthy children in Boston, in Caracas, Venezuela, and in Qin Pu, China. New England Journal of Medicine 323, 285-289.

Lidbeck A \& Nord CE (1991) Lactobacilli in relation to human ecology and antimicrobial therapy. International Journal of Tissue Reactions 13, 115-122.

Lowry OH, Rosebrough NJ, Farr AL \& Randall RJ (1951) Protein measurement with the Folin phenol reagent. Journal of Biological Chemistry 193, 265-275.

Maisonneuve S, Ouriet MF \& Duval-Iflah Y (2000) Effects of yogurt intake on plasmid transfer and colonization with transconjugants in the digestive tract of mice associated with human fecal flora. FEMS Microbiology and Ecology 31, $241-248$.

Maisonneuve S, Ouriet MF \& Duval-Iflah Y (2001) Comparison of yogurt, heat treated yogurt, milk and lactose effects on plasmid dissemination in gnotobiotic mice. Antonie van Leeuwenhoek 79, 199-207.
Marteau P, Flourie B, Pochart P, Chastang C, Desjeux JF \& Rambaud JC (1990) Effect of the microbial lactase (EC 3.2.1.23) activity in yoghurt on the intestinal absorption of lactose: an in vivo study in lactase-deficient humans. British Journal of Nutrition 64, 71-79.

Pedrosa MC, Golner BB, Goldin BR, Barakat S, Dallal GE \& Russell RM (1995) Survival of yogurt-containing organisms and Lactobacillus gasseri (ADH) and their effect on bacterial enzyme activity in the gastrointestinal tract of healthy and hypochlorhydric elderly subjects. American Journal of Clinical Nutrition 61, 353-359.

Pochart P, Dewit O, Desjeux JF \& Bourlioux P (1989) Viable starter culture, beta-galactosidase activity, and lactose in duodenum after yogurt ingestion in lactase-deficient humans. American Journal of Clinical Nutrition 49, 828-831.

Raibaud P, Dickinson AB, Sacquet E, Charlier H \& Mocquot G (1966) La microflore du tube digestif du rat. I. Techniques d'étude et milieux de culture proposés (Microflora of the rat digestive tract. I. Techniques and growth media). Annales de l'Institut Pasteur (Paris) 110, 568-590.

Shermak MA, Saavedra JM, Jackson TL, Huang SS, Bayless TM \& Perman JA (1995) Effect of yogurt on symptoms and kinetics of hydrogen production in lactose-malabsorbing children. American Journal of Clinical Nutrition 62, 1003-1006.

Viljanen P \& Boratynski J (1991) The susceptibility of conjugative resistance transfer in Gram-negative bacteria to physicochemical and biochemical agents. FEMS Microbiology Reviews $\mathbf{8 8}, 43-54$.

Walker DK \& Gilliland SE (1993) Relationship among bile tolerance, bile salt deconjugation, and assimilation of cholesterol by Lactobacillus acidophilus. Journal of Dairy Science 76, 956-961. 